\title{
Photorefractive Multiple Quantum Well Waveguide as a Bragg Reflector
}

\author{
M. WichtoWski* And E. WeinerT-RĄCzKA \\ Institute of Electronics, Telecommunication and Informatics \\ Electrical Engineering Department, Technical University of Szczecin \\ al. Piastów 17, 70-310 Szczecin, Poland \\ An interaction between the signal wave propagating in a planar wave- \\ guide and a photorefractive grating is analysed. The waveguide contains an \\ AlGaAs/GaAs multiple quantum well guiding layer biased with an external \\ electric field applied along the wells plane. The grating is formed by two ex- \\ ternal beams interfering in the multiple quantum well layer. The dependence \\ of the steady-state grating properties on the external waves parameters and \\ applied electric field intensity is presented. Possible application of the grating \\ as an optically controlled Bragg reflector with memory is analysed.
}

PACS numbers: 42.65.-k, 42.65.Pc

\section{Introduction}

A photorefractive grating in semi-insulating multiple quantum well waveguide can be used as an optically induced mode coupling element with memory [1]. The grating, created by two mutually coherent waves interfering in a nonlinear material, can be used for the same purposes as stable or electro-optically induced grating, but it can be tuned in a real time by varying parameters of the interfering waves. The grating does not require a permanent presence of the writing beams, which are necessary only for writing, refreshing, or erasing the grating. The amplitude of the grating can be high enough to influence signals propagating in the waveguide. The signal waves with low frequency do not destroy the grating and the system may have applications as an all-optical switching element with memory.

Here we analyse properties of the photorefractive grating designed to serve as a Bragg reflector. The grating is induced in a single-mode planar waveguide

* corresponding author; e-mail: marekw ${ }^{0} \mathrm{ps} . \mathrm{pl}$ 
containing semi-insulating multiple quantum well (MQW) structure as a guiding layer. The MQW structure operates in so-called transverse Franz-Keldysh geometry [2] with an external electric field applied along the quantum well planes as shown in Fig. 1.

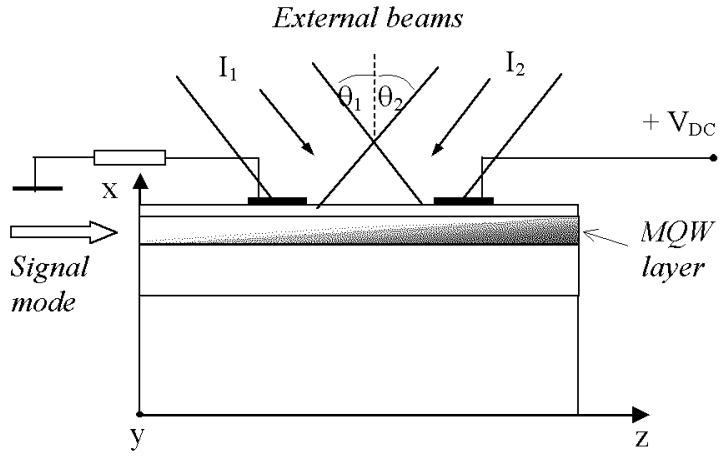

Fig. 1. Photorefractive MQW waveguide geometry.

The grating is created by two mutually coherent external beams of the same polarization falling on the surface of the sample. The interference between the beams overlapping in the MQW layer gives the intensity pattern described by

$$
I(x, z)=I_{0} \exp (-\alpha x)[1+m \cos (K z)],
$$

where $I_{0}=I_{1}+I_{2}$ denotes a total intensity of the beams at the entrance to the MQW layer, $\alpha$ is the absorption coefficient, $m$ is the modulation depth (fringe visibility) given by $m=2\left(I_{1} I_{2}\right)^{1 / 2} / I_{0}$. The grating constant $K$ depends on incidence angles and for $\theta_{1}=\theta_{2}=\theta$ is given by

$$
K=2 n_{\mathrm{p}} k_{\mathrm{ex}} \sin \theta,
$$

where $n_{\mathrm{p}}$ denotes the refractive index of the outer medium and $k_{\mathrm{ex}}$ - the wave vector of the external beams. A high frequency light creates electron-hole pairs by direct interband transitions. Free carriers move and finally recombine to the donor traps mainly in the dark regions of the interference pattern, creating a spatially modulated charge distribution. Non-uniform charge distribution builds up a space-charge electric field which changes the refractive index through the electro-optic effect $[3,4]$.

\section{Properties of the waveguide}

The structure of the waveguide is shown in Fig. 2. The photorefractive MQW layer contains sixty periods of $7.5 \mathrm{~nm}$ thick GaAs wells and $10 \mathrm{~nm}$ thick $\mathrm{Al}_{0.3} \mathrm{Ga}_{0.7} \mathrm{As}$ barriers. The guiding layer is sandwiched between two $\mathrm{Al}_{0.24} \mathrm{Ga}_{0.76} \mathrm{As}$ covers and deposited on the GaAs substrate. A thin protecting GaAs layer is placed 
on the cover. The wavelengths of interacting beams are chosen according to the absorption and electrorefraction spectra of MQW [1]. The guided modes reading the grating are assumed to have the wavelength about $850 \mathrm{~nm}$ to obtain relatively low absorption and possibly high electro-optic coefficient. The structure is designed to form a single-mode waveguide for such wavelength. The effective refractive index $N_{\text {eff }}$ and transverse field distribution of the fundamental $\mathbf{T E}_{0}$ mode (presented in Fig. 3) are calculated using a commercial software [5] based on transmission matrix method.

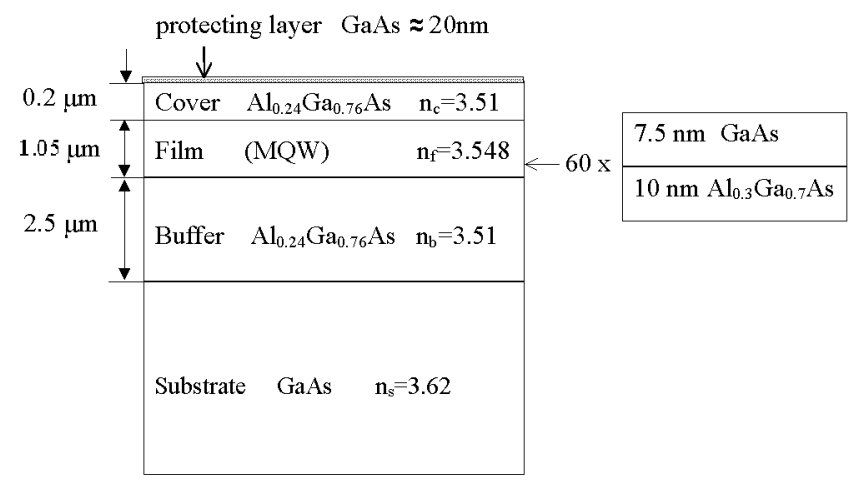

Fig. 2. Cross-section of the waveguide.

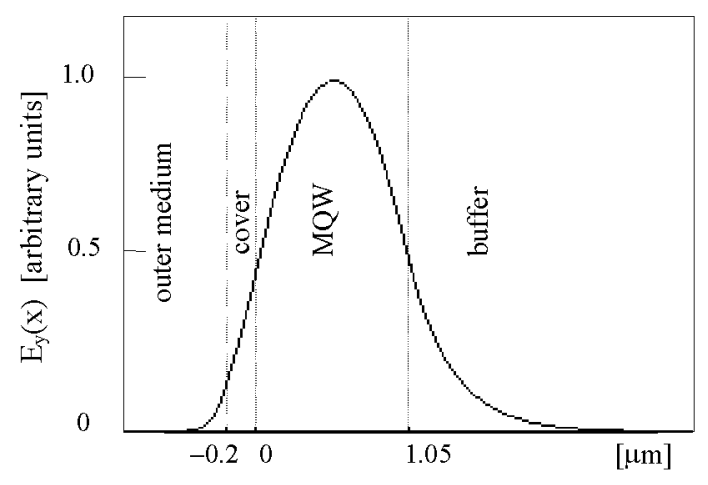

Fig. 3. The field profile of the fundamental $\mathrm{TE}_{0}$ mode.

An average refractive index of MQW structure can be determined from the formula $[6]$ :

$$
n_{\mathrm{f}}=\left(\frac{n_{\mathrm{w}}^{2} L_{\mathrm{w}}+n_{\mathrm{b}}^{2} L_{\mathrm{b}}}{L_{\mathrm{w}}+L_{\mathrm{b}}}\right)^{1 / 2},
$$

where $n_{\mathrm{w}}$ and $n_{\mathrm{b}}$ denote the refractive indices and $L_{\mathrm{w}}, L_{\mathrm{b}}$ the thicknesses of wells and barriers, respectively. The field distribution and the effective refractive 
index obtained for a uniform layer of $n_{\mathrm{f}}$ placed between the covers are practically identical as for the full structure.

The grating constant necessary to obtain Bragg reflection $[7,8]$ is

$$
K=2 k_{\mathrm{G}} N_{\mathrm{eff}} \cos \varphi,
$$

where $k_{\mathrm{G}}=2 \pi / \lambda_{\mathrm{G}}$ is the free-space wave vector of the signal beam, $N_{\text {eff }}$ is the effective refractive index of the signal mode and $\varphi$ is the signal beam incidence angle. The required grating constant is so large (for a normal incidence $K=$ $5.232 \times 10^{5} \mathrm{~cm}^{-1}$ which corresponds to space period $A=2 \pi / K=0.12 \mu \mathrm{m}$ ) that the external beams have to enter the sample by a prism with the refraction index satisfying the following condition:

$$
n_{\mathrm{p}}=\frac{\lambda_{\mathrm{ex}}}{\lambda_{\mathrm{G}}} N_{\mathrm{eff}} \frac{\cos \phi}{\sin \theta}
$$

where $\theta$ is the incidence angle of external waves. For the backward reflection $(\phi=0)$ the prism should have the refractive index higher than 2.63 , which can be obtained using materials like rutyl $\left(\mathrm{TiO}_{2}\right)$ or one of the semiconductors transparent for the external beams wavelength (e.g. GaP).

\section{Space-charge electric field}

The amplitude of the grating depends on the amplitude of the space charge electric field pattern which can be obtained from one-dimensional transport equations [9]. If thermal excitation and direct recombination of the carriers are not included, the equations for MQW planes perpendicular to the $x$-axis and the electric field applied along the $z$-axis take the form [1]:

$$
\begin{aligned}
& \frac{\partial n_{\mathrm{e}}}{\partial t}=\frac{\alpha}{h \nu} I-\gamma_{\mathrm{e}} n_{\mathrm{e}} N_{\mathrm{D}}^{+}+\frac{1}{e} \frac{\partial j_{\mathrm{e}}}{\partial z} \\
& \frac{\partial n_{\mathrm{h}}}{\partial t}=\frac{\alpha}{h \nu} I-\gamma_{\mathrm{h}} n_{\mathrm{h}}\left(N_{\mathrm{D}}-N_{\mathrm{D}}^{+}\right)-\frac{1}{e} \frac{\partial j_{\mathrm{h}}}{\partial z} \\
& j_{\mathrm{e}}=e \mu_{\mathrm{e}} n_{\mathrm{e}} E+\mu_{\mathrm{e}} k_{\mathrm{B}} T \frac{\partial n_{\mathrm{e}}}{\partial z} \\
& j_{\mathrm{h}}=e \mu_{\mathrm{h}} n_{\mathrm{h}} E-\mu_{\mathrm{h}} k_{\mathrm{B}} T \frac{\partial n_{\mathrm{h}}}{\partial z} \\
& \frac{\partial N_{\mathrm{D}}^{+}}{\partial t}=\gamma_{\mathrm{h}} n_{\mathrm{h}}\left(N_{\mathrm{D}}-N_{\mathrm{D}}^{+}\right)-\gamma_{\mathrm{e}} n_{\mathrm{e}} N_{\mathrm{D}}^{+}, \\
& \frac{\partial E}{\partial z}=\frac{e}{\varepsilon \varepsilon_{0}}\left(N_{\mathrm{D}}^{+}+n_{\mathrm{h}}-n_{\mathrm{e}}-N_{\mathrm{A}}\right)
\end{aligned}
$$

where $n_{\mathrm{e}}$ denotes the free electron and $n_{\mathrm{h}}$ - the free hole concentration, $N_{\mathrm{D}}$ donor, $N_{\mathrm{D}}^{+}$- ionised donor, $N_{\mathrm{A}}$ - acceptor concentrations, $j_{\mathrm{e}}$ - the electronic and $j_{\mathrm{h}}$ - the hole current densities, $E$ - the total electric field ( $E=E_{\mathrm{a}}+E_{\mathrm{sc}}$, where $E_{\mathrm{sc}}$ is a space-charge field and $E_{\mathrm{a}}$ is an external field), $I$ - the light intensity, 
$\gamma_{\mathrm{e}}$ and $\gamma_{\mathrm{h}}$ - electron and hole recombination constants, $\mu_{\mathrm{e}}$ - electron and $\mu_{\mathrm{h}}-$ hole mobilities along the quantum wells, $\varepsilon_{0}$ - the vacuum permittivity, $\varepsilon$ - the effective dielectric constant of MQW structure, $e$ - the elementary charge value, $k_{\mathrm{B}}$ - the Boltzmann constant and $T$ - the absolute temperature. The transverse carrier mobility is neglected due to the high difference between longitudinal and transverse mobilities characteristic of MQW structure.

Under the steady-state conditions and for light intensity given by (1) the space charge field can be presented in a form of the Fourier series [1, 10]:

$$
\begin{aligned}
E(z) & =E_{\mathrm{a}}+m E_{1} \exp (\mathrm{i} K z)+m^{2} E_{2} \exp (\mathrm{i} 2 K z) \\
& +m^{3} E_{3} \exp (\mathrm{i} 3 K z)+\ldots
\end{aligned}
$$

where $E_{1}, E_{2}, E_{3}$, etc. are generally complex numbers due to the arbitrary phase shift with respect to the interference pattern. Modules of the first three Fourier components as functions of the applied electric field are plotted in Fig. 4. For the considered grating constant the amplitude of the first harmonic is nearly two orders of magnitude greater than the second and about three orders of magnitude greater than the third one. Therefore the influence of the higher order terms on the propagation of guided modes can be neglected.

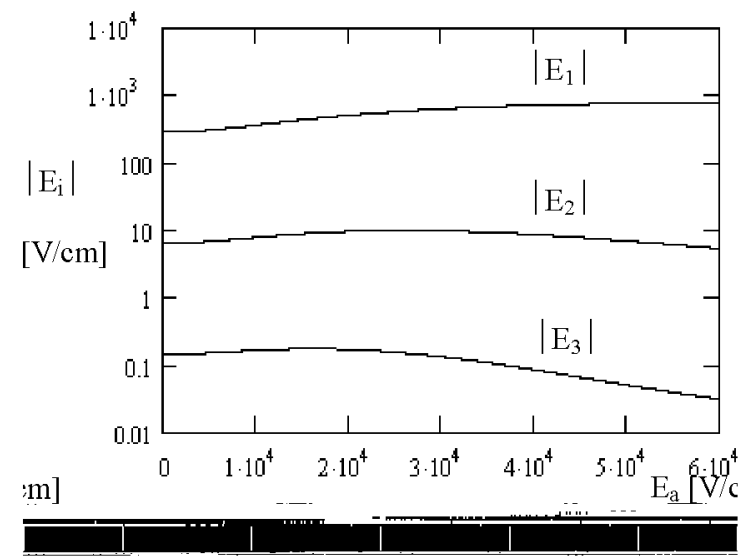

Fig. 4. The first three harmonic components amplitudes versus the external electric field for $\Lambda=0.12 \mu \mathrm{m}$ and $N_{\mathrm{D}}=2 \times 10^{18} \mathrm{~cm}^{-3}$.

The dependence of the first Fourier amplitude on the applied electric field for various trap concentrations is shown in Fig. 5. Because of the small grating space period a high trap density is necessary to obtain the increase in the space-charge field amplitude with an external electric field. For example for trap density of $2 \times 10^{18} \mathrm{~cm}^{-3}$ the applied field of $10 \mathrm{kV} / \mathrm{cm}$ can increase the space charge field from $280 \mathrm{~V} / \mathrm{cm}$ to about $350 \mathrm{~V} / \mathrm{cm}$. The phase shift between the first Fourier component of the space charge field and the light pattern as a function of an external electric field is depicted in Fig. 6. Without the applied field diffusion plays 


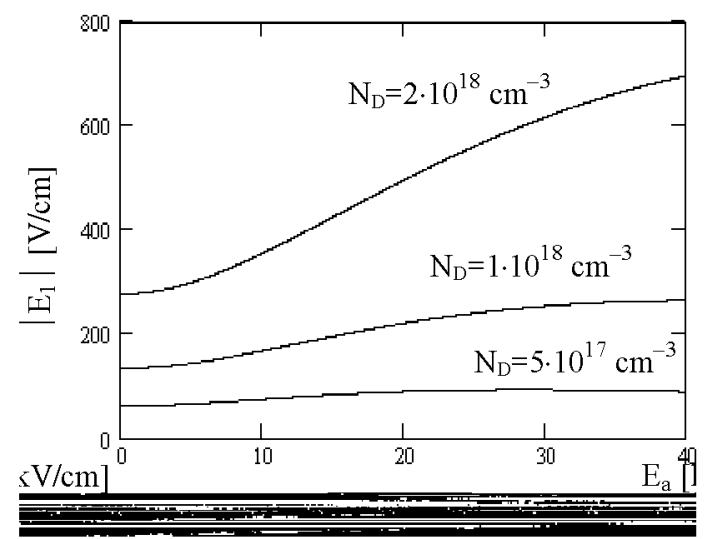

Fig. 5. The first order amplitude $E_{1}$ versus an external field for three different traps densities.

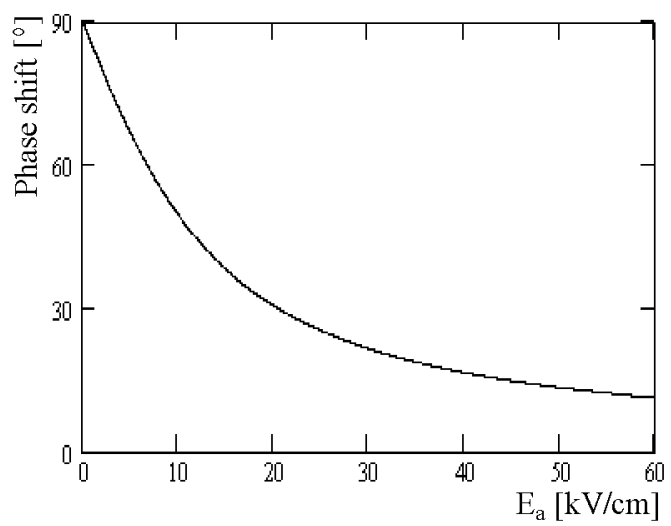

Fig. 6. The phase shift between the space charge field and the light pattern versus an external field (phase shift is independent of the trap density).

the most important role in carrier transport and the space-charge field is shifted by $90 \mathrm{deg}$ with respect to the interference pattern [11] ( $E_{1}$ is purely imaginary). For high intensities of the applied field drift mechanism becomes more important and phase shift decreases in the considered case $\left(E_{\mathrm{a}}=10 \mathrm{kV} / \mathrm{cm}\right)$ to about $50 \mathrm{deg}$.

Time evolution of space charge field was found by numerical solution of Eqs. (5)-(10) with the Runge-Kutta method. The dependence of space charge field amplitude on the absorbed energy of the light beams with constant intensity is shown in Fig. 7. Time evolution of the space charge field driven by Gaussian pulses is presented in Fig. 8. The first pulse, consisting of two interfering beams, records the grating and the second one, being a single uniform beam, erases it. The grating lifetime after switching off the external illumination depends mainly on the dark conductivity of the material and for semi-insulating MQW structure can be much longer than the recording time. 


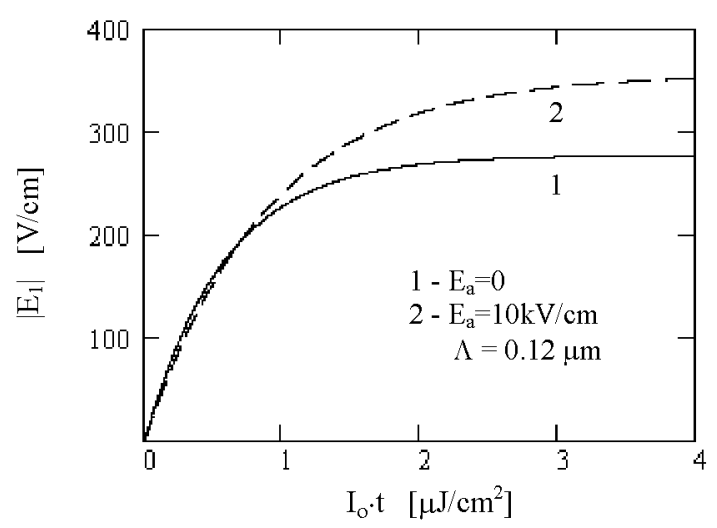

Fig. 7. The amplitude $E_{1}$ as a function of external beams energy for the sample with no external electric field and for applied field of $10 \mathrm{kV} / \mathrm{cm}$.

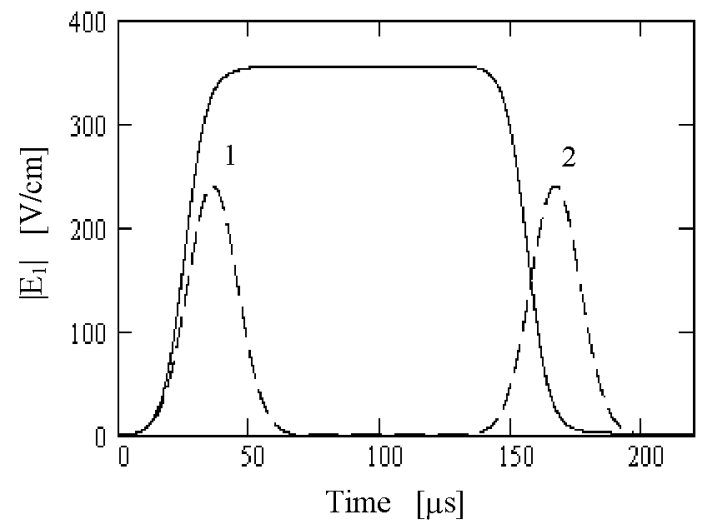

Fig. 8. Time evolution of the first Fourier component of space charge field for two short pulses of light with the intensity amplitude $I_{0}=0.1 \mathrm{~W} / \mathrm{cm}^{2}$. Pulse 1 is writing the grating and pulse 2 is erasing it.

\section{Efficiency of the grating as a Bragg reflector}

A sum of the applied electric field and spatially modulated space-charge field induce the refractive index change, which can be described by a quadratic electro-optic effect. The refractive index change in MQW structure can be approximated by [4]:

$$
\Delta n(\lambda)=(-1 / 2) n_{\mathrm{f}}^{3} s(\lambda) E^{2},
$$

where $n_{\mathrm{f}}$ is an average index of the MQW structure, $s(\lambda)$ is the quadratic electro-optic coefficient and $E=E_{\mathrm{a}}+E_{\mathrm{sc}}$ is the total electric field. The electro-optic coefficient for wavelength of $850 \mathrm{~nm}$ is in the range of $2 \times 10^{-13} \mathrm{~cm}^{2} / \mathrm{V}^{2}$ [1]. For the electric field $E_{\mathrm{sc}}(z) \cong E_{1}(z)=\left|E_{1}\right| \cos \left(K z+\phi_{1}\right)$ the refractive index change can be written as sum of three elements 


$$
\Delta n(\lambda, z)=\Delta n_{0}(\lambda)+\Delta n_{1}(\lambda) \cos \left(K z+\phi_{1}\right)+\Delta n_{2}(\lambda) \cos \left(2 K z+\phi_{2}\right),
$$

where $\Delta n_{0}(\lambda)$ is proportional to the average electric field $E_{\mathrm{a}}^{2}+(1 / 2)\left|E_{1}\right|^{2}, \Delta n_{1}(\lambda)$ is proportional to $E_{a}\left|E_{1}\right|$ and the last component $\Delta n_{2}(\lambda)$ to $(1 / 2)\left|E_{1}\right|^{2}$. For $E_{1}$ much smaller than $E_{\mathrm{a}}$ the last term can be neglected and the refractive index grating amplitude is

$$
\Delta n_{1}=-n_{\mathrm{f}}^{3} s E_{\mathrm{a}}\left|E_{1}\right|
$$

The dependence of the refractive index amplitude on the applied electric field for different trap concentrations is shown in Fig. 9.

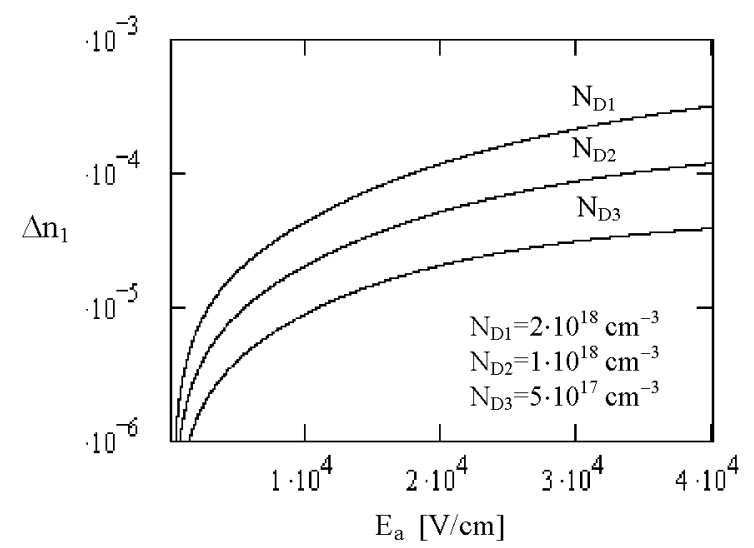

Fig. 9. The refractive index grating amplitude versus an applied electric field for the grating space period of $0.12 \mu \mathrm{m}$.

For the normal incidence of the signal wave on the periodic structure the amplitude of the reflected wave, in the case of the grating constant perfectly matched to the propagation vectors difference, is given by [7]:

$$
A_{\mathrm{B}}(0)=A_{0} \tanh (\chi L),
$$

where $A_{0}$ denotes the amplitude of the incident mode for $z=0, A_{\mathrm{B}}(0)$ - the amplitude of the backward mode for $z=0$ and $L$ - the length of the grating. The coupling coefficient $\chi$, describing interaction between the signal mode and the reflected mode, can be calculated from the following formula:

$$
\chi=\Delta n_{1} \frac{k_{\mathrm{G}}^{2} n_{\mathrm{f}}}{K} \frac{\int_{0}^{h}\left|E_{y}(x)\right|^{2} \mathrm{~d} x}{\int_{-\infty}^{\infty}\left|E_{y}(x)\right|^{2} \mathrm{~d} x},
$$

where $k_{\mathrm{G}}$ is the free-space wave vector of the signal beam, $h$ is a thickness of the MQW layer and $E_{y}(x)$ is a transverse field distribution of the guided mode. The amplitude of the refractive index grating calculated for the parameters listed in Tables I and II is $\Delta n_{1} \approx 3 \times 10^{-5}$. The coupling coefficient is directly proportional to the refractive index amplitude and for the described waveguide is about $\chi \approx$ $1.2 \mathrm{~cm}^{-1}$. Dependence of the reflection coefficient $R=A_{\mathrm{B}}(0) / A_{0}$ on the length of such a grating is plotted in Fig. 10. 
TABLE I

Parameters used in study of the space-charge field amplitudes.

\begin{tabular}{l|l}
\hline \hline$\mu_{\mathrm{e}}=5000 \mathrm{~cm}^{2} /(\mathrm{V} \mathrm{s})$ & the electron mobility \\
$\mu_{\mathrm{h}}=300 \mathrm{~cm}^{2} /(\mathrm{V} \mathrm{s})$ & the hole mobility \\
$\gamma_{\mathrm{e}}=\gamma_{\mathrm{h}}=10^{7} \mathrm{~cm}^{3} / \mathrm{s}$ & the trapping coefficient* \\
$m \cong 1$ & the fringes visibility \\
$n_{\mathrm{f}}=3.548$ & the average refractive index of the MQW layer \\
$\varepsilon=n_{\mathrm{f}}^{2}=12.586$ & the effective dielectric constant of MQW layer \\
$r=N_{\mathrm{A}} / N_{\mathrm{D}}=0.5$ & the compensation ratio of donors traps \\
$K=5.232 \times 10^{5} \mathrm{~cm}^{-1}$ & the grating constant \\
$\Lambda=0.12 \mu \mathrm{m}$ & the fringe spacing \\
\hline
\end{tabular}

${ }^{*}$ Values of carrier mobilities and trapping coefficient were derived from [10].

TABLE II

Parameters used in study of grating amplitude and reflection coefficient.

\begin{tabular}{l|l}
\hline \hline$s=2 \times 10^{-13} \mathrm{~cm}^{2} / \mathrm{V}^{2}$ & the quadratic electro-optic coefficient \\
$E_{\mathrm{a}}=10 \mathrm{kV} / \mathrm{cm}$ & the external electric field \\
$N_{\mathrm{D}}=2 \times 10^{18} \mathrm{~cm}^{-3}$ & the donors trap density \\
$r=N_{\mathrm{A}} / N_{\mathrm{D}}=0.5$ & the compensation ratio of impurities \\
$\lambda_{\mathrm{G}}=850 \mathrm{~nm}$ & the wavelength of signal wave \\
$\lambda_{\mathrm{ex}} \approx 630 \mathrm{~nm}$ & the wavelength of writing waves \\
$\alpha \cong 10^{4} \mathrm{~cm}^{-1}$ & the absorption coefficient of the writing wave \\
$I_{0}=0.1 \mathrm{~W} / \mathrm{cm}^{2}$ & the total average intensity of the writing beams \\
$N_{\mathrm{eff}}=3.54$ & the effective refractive index for TE $\mathrm{TE}_{0}$ mode \\
$h=1.05 \mu \mathrm{m}$ & the MQW thickness
\end{tabular}

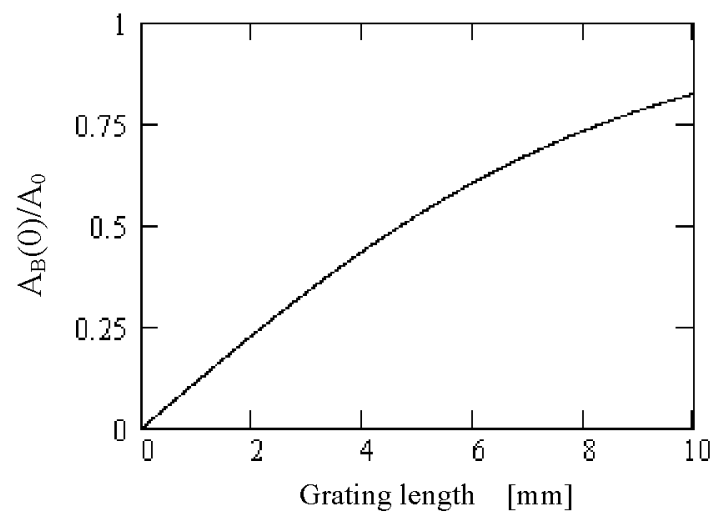

Fig. 10. The normalised amplitude of the reflected mode, $A_{\mathrm{B}}(0) / A_{0}$, versus the grating length. 


\section{Conclusions}

The results of calculations show that the photorefractive grating in semi-insulating MQW waveguide operating with the electric field of $10 \mathrm{kV} / \mathrm{cm}$ applied along the QW planes can be used as an optically controlled Bragg reflector with memory. The amplitude and the space period of the grating depend on the external waves parameters and can be tuned during the work of the device. Short grating space period necessary to obtain backward reflection require high concentration of traps in MQW structure (about $2 \times 10^{18} \mathrm{~cm}^{-3}$ ) which can be obtained by proton implantation [12].

\section{References}

[1] E. Weinert-Rączka, M. Wichtowski, A. Ziółkowski, G. Staroń, Acta Phys. Pol. A $103, \ldots(2003)$.

[2] A.M. Glass, D.D. Nolte, H.D. Olson, G.E. Doran, D.S. Chemla, W.H. Knox, Opt. Lett. 15, 264 (1990).

[3] D.D. Nolte, D.H. Olson, G.E. Doran, W.H. Knox, A.M. Glass, J. Opt. Soc. Am. $B$ 7, 2217 (1990).

[4] E. Weinert-Rączka, R. Iwanow, Acta Phys. Pol. A 99, 175 (2001).

[5] L. Leine, C. Wächter, software Tramax, Jena (1999).

[6] S. Ohke, T. Umeda, Y. Cho, Opt. Commun. 56, 239 (1985).

[7] J. Petykiewicz, Podstawy fizyczne optyki scalonej, PWN, Warszawa 1989 (in Polish) or H. Kogelnik, Topics in Applied Physics 7, Springer-Verlag, New York 1975.

[8] H. Stoll, A. Yariv, Opt. Commun. 8, 5 (1973).

[9] Q. Wang, R.M. Brubaker, D.D. Nolte, M.R. Melloch, J. Opt. Soc. Am. B 9, 1626 (1992).

[10] L.F. Magaňa, F. Agulló-López, M. Carrascosa, J. Opt. Soc. Am. B 11, 1651 (1994).

[11] J.P. Huignard, P. Günter, Photorefractive Materials and Their Applications I, Springer-Verlag, Berlin 1988.

[12] D.D. Nolte, J. Appl. Phys. 85, 6259 (1999). 